\title{
Estenose pulmonar em duas cadelas: Relato de caso
}

\author{
Pulmonary stenosis in two female dogs: Case report \\ Estenosis pulmonar en dos perras: Reporte de caso
}

Recebido: 18/05/2021 | Revisado: 30/05/2021 | Aceito: 06/06/2021 | Publicado: 20/06/2021

\author{
Mayara Cristtine Ramos \\ ORCID: https://orcid.org/0000-0002-8665-9898 \\ Universidade Federal de Pelotas, Brasil \\ E-mail: mayaracramos@outlook.com.br \\ Eduarda Machado dos Santos \\ ORCID: https://orcid.org/0000-0003-2384-3345 \\ Universidade Federal de Pelotas, Brasil \\ E-mail: dudarecart@msn.com \\ Eduarda Aléxia Nunes Louzada Dias Cavalcanti \\ ORCID: https://orcid.org/0000-0002-4898-7428 \\ Universidade Federal de Pelotas, Brasil \\ E-mail: nuneslouzadadias@gmail.com \\ Mariana Wilhelm Magnabosco \\ ORCID: https://orcid.org/0000-0003-3214-5370 \\ Universidade Federal de Pelotas, Brasil \\ E-mail: mwmagnabosco@gmail.com \\ Paula Priscila Correia Costa \\ ORCID: https://orcid.org/0000-0001-6421-0689 \\ Universidade Federal de Pelotas, Brasil \\ E-mail: paulapriscilamv@yahoo.com.br \\ Guilherme Albuquerque de Oliveira Cavalcanti \\ ORCID: https://orcid.org/0000-0001-8340-9087 \\ Universidade Federal de Pelotas, Brasil \\ E-mail: guialbuquerque@yahoo.com
}

\begin{abstract}
Resumo
A estenose pulmonar é uma doença congênita que causa obstrução parcial do fluxo sanguíneo entre o ventrículo direito e a artéria pulmonar, sendo encontrada principalmente em animais jovens e machos. Este trabalho relata os casos de duas cadelas das raças Spitz Alemão e Yorkshire Terrier, com 8 meses e 9 anos, respectivamente. Os animais foram submetidos a radiografias latero-lateriais e ventro-dorsais torácicas que evidenciaram aumento cardíaco direito e dilatação do tronco da artéria pulmonar. Também foram submetidas ao exame ecocardiográfico que demonstrou valvas pulmonares com função e morfologia alterada e dilatação pós-estenótica, com fluxo de velocidade aumentada e turbulento na região dessa valva. Após o diagnóstico, estabeleceu-se tratamento clínico com o uso de vasodilatadores, diuréticos, inibidor da fosfodiesterase e $\beta$-bloqueador dependendo da necessidade de cada animal, sendo recomendada a avaliação clínica periódica das pacientes e realização de exames complementares para acompanhamento dos casos. O tratamento estabelecido se mostrou satisfatório, devido a melhora dos sinais clínicos.
\end{abstract} Palavras-chave: Cães; Cardiopatia; Doença congênita.

\begin{abstract}
Pulmonary stenosis is a congenital disease that causes partial obstruction of blood flow between the right ventricle and the pulmonary artery and is found mainly in young and male animals. This work reports the cases of two female dogs of the German Spitz and Yorkshire Terrier breeds, aged 8 months and 9 years respectively. The animals were submitted to lateral and ventrodorsal chest X-rays, which showed right cardiac enlargement and dilation of the pulmonary artery trunk. They were also submitted to an echocardiographic examination that demonstrated pulmonary valves with altered function and morphology and post-stenotic dilation with increased velocity flow, as well as turbulent in the region of this valve. After diagnosis, clinical treatment was established with the use of vasodilators, diuretics, phosphodiesterase inhibitor and $\beta$-blocker depending on the needs of each animal, with periodic clinical evaluation of patients and complementary exams to monitor cases being recommended. The established treatment proved to be satisfactory, due to the improvement of clinical signs.
\end{abstract}

Keywords: Dogs; Heart disease; Congenital disease. 


\begin{abstract}
Resumen
La estenosis pulmonar es una enfermedad congénita que causa una obstrucción parcial del flujo sanguíneo entre el ventrículo derecho y la arteria pulmonar y se encuentra principalmente en animales jóvenes y machos. Este trabajo informa los casos de dos perras de las razas German Spitz y Yorkshire Terrier, de 8 meses y 9 años respectivamente. Los animales fueron sometidos a radiografías de tórax laterolateral y ventrodorsal, que mostraron agrandamiento cardíaco derecho y dilatación del tronco de la arteria pulmonar. También fueron sometidos a un examen ecocardiográfico que evidenció válvulas pulmonares con función y morfología alterada y dilatación postestenótica con aumento de velocidad de flujo, así como turbulento en la región de esta válvula. Tras el diagnóstico, se estableció el tratamiento clínico con el uso de vasodilatadores, diuréticos, inhibidor de la fosfodiesterasa y bloqueador $\beta$ en función de las necesidades de cada animal, recomendándose la evaluación clínica periódica de los pacientes y exámenes complementarios para seguimiento de casos. El tratamiento establecido resultó satisfactorio, debido a la mejoría de los signos clínicos.
\end{abstract}

Palabras clave: Perros; Cardiopatía; Enfermedad congénita.

\title{
1. Introdução
}

As cardiopatias congênitas são incomuns em cães e caracterizam-se por defeitos morfológicos no coração ou grandes vasos, presentes desde o nascimento do indivíduo. Essas anormalidades surgem durante o desenvolvimento embrionário fetal (Macdonald, 2006; Lázaro et al., 2015). Dentre as cardiopatias congênitas caninas, a estenose pulmonar (EP) está entre as três mais frequentes, afetando de 18 a 34\% de todos os pacientes com doença cardíaca congênita. As outras duas mais frequentes são a persistência do ducto arterioso e a estenose subaórtica (Buchanan, 1992; Serrano, 2012; Brambilla et al., 2020).

A EP é uma obstrução do trato de saída do ventrículo direito devido a um estreitamento anormal da valva ou da própria artéria pulmonar (Sisson, 2015; Santos, 2019). Ela pode ocorrer em qualquer parte do trato de saída do ventrículo direito, podendo ser classificado como valvar, subvalvar ou supravalvar, sendo possíveis múltiplos locais de estenose no mesmo animal (Bussadori et al., 2000; Bussadori et al., 2001; Macdonald, 2006; Boon, 2011; Locatelli et al., 2013; Estrada \& Maisenbacher, 2015; Santos, 2019). A estenose do tipo valvar é a mais relatada em cães, essa malformação pode ser resultado de uma combinação de hipoplasia, espessamento/separação incompleta comissural e assimetria dos folhetos valvares, afetando a via de saída sanguínea do ventrículo direito para os pulmões (Macdonald, 2006; Schrope, 2007; Filho, 2011; Kobayashi, 2014; Lázaro et al., 2015).

Cães da raça Beagle possuem um padrão hereditário poligênico de EP, sendo a única raça com padrão hereditário comprovado (Detweiler \& Patterson, 1965; Santos, 2019), entretanto, as raças braquiocefálias, Bulldog inglês e Bulldog francês, foram as mais acometidas por essa afecção, segundo um estudo realizado por Brambilla et al. (2020). Cães das raças Pinchers miniatura, Cocker Spaniel, Boxer, Yorkshire Terrier, Golden Retriever e Rottweiler, entre outras, também podem apresentar a EP (Brambilla et al., 2020).

Os sinais clínicos variam de pacientes assintomáticos a pacientes graves, sendo os primeiros identificados apenas pela presença de sopro sistólico durante ausculta cardíaca e com encaminhamento a exames de imagem. Animais com a doença em nível mais avançado apresentam sintomatologia de insuficiência cardíaca congestiva direita, como, ascite, hepatomegalia acompanhado ou não de esplenomegalia, intolerância a exercício e em casos mais graves até sincope e morte (Oliveira, 2015; Sousa et al., 2020).

O diagnóstico para EP é dado através da análise do histórico do animal, aliado aos sinais clínicos e exames complementares, dos quais se recomendam eletrocardiografia, radiografia torácica, ecocardiografia, angiografia e cateterismo cardíaco (Ristic et al., 2001, Sousa et al., 2020). Seu tratamento depende da gravidade da doença, quadros leves, geralmente não necessitam de tratamento, porém é recomendado a avaliação periódica do paciente. Em casos graves, o tratamento cirúrgico é recomendado (Oyama et al., 2005).

Neste trabalho, objetivou-se relatar os casos clínicos de duas cadelas das raças Spitz Alemão e Yorkshire Terrier, atendidos no Hospital de Clínicas Veterinárias da Universidade Federal de Pelotas (UFPel) apresentado EP. 


\section{Relato de caso}

\section{Caso 1:}

Uma cadela da raça Yorkshire Terrier de 9 anos de idade, com histórico de tosse e cianose quando submetida a estresse, apresentou no exame físico sopro cardíaco. Com isso, a paciente foi encaminhada para a realização de exame radiográfico torácico e exame ecocardiográfico no Laboratório de Diagnóstico por Imagem e Cardiologia (LADIC) da UFPel.

\section{Caso 2:}

Uma cadela da raça Spitz Alemão, com 8 meses de idade e histórico de ingestão da planta Espada de São Jorge (Dracaena trifasciata). Observou-se na paciente sialorréia, no exame físico notou-se, durante a auscultação, a presença de sopro cardíaco de grau IV em foco pulmonar e ruídos respiratórios. Sendo assim, a paciente foi encaminhada para a realização de exame radiográfico torácico e exame ecocardiográfico no LADIC da UFPel.

\section{Resultados e Discussão}

A EP foi diagnosticada aos 9 anos do paciente do Caso 1, e aos 8 meses do paciente do Caso 2. De acordo com Ware (2015), esta doença ocorre com maior frequência em animais jovens, com idade entre 5 e 16 meses, dessa forma, apenas o Caso 2 corrobora com o descrito. Em relação ao sexo, esta afecção tem uma maior prevalência em machos (Locatelli et al., 2013; Santos, 2019), entretanto neste trabalho, é relatada em duas fêmeas.

Segundo Bradley (2013), a realização de exame radiográfico é indicada para avaliação do sistema cardiovascular a fim de investigar um sopro detectado ao exame físico, como o apresentado por ambas pacientes. O sopro cardíaco na EP, é decorrente da passagem turbulenta do sangue pela área de estenose durante a sístole ventricular (Serrano, 2012).

Através da avaliação radiográfica torácica observou-se aumento do tronco da artéria pulmonar (Figura 1 e Figura 2), este sinal é descrito por Zani et al. (2004) como resultante do fluxo turbulento e de alta velocidade crônico ocasionando uma dilatação pós-estenótica (Kittleson, 1998; Fox, 1999; Serrano, 2012). Além deste, visualizou-se na paciente do Caso 1 aumento global da silhueta cardíaca destacada em lado direito, opacidade pulmonar de padrão misto bronco-alveolar e diminuição do lúmen traqueal em porção cervical (Figura 1), na paciente do Caso 2 verificou-se padrão pulmonar alveolar (Figura 2).

Figura 1: Projeções radiográficas lateral direita (A) e ventro-dorsal (B) de uma cadela da raça Yorkshire Terrier (Caso 1), evidenciando aumento global da silhueta cardíaca com aumento acentuado em região do tronco da artéria pulmonar (seta branca), e opacidade pulmonar de padrão bronco-alveolar.

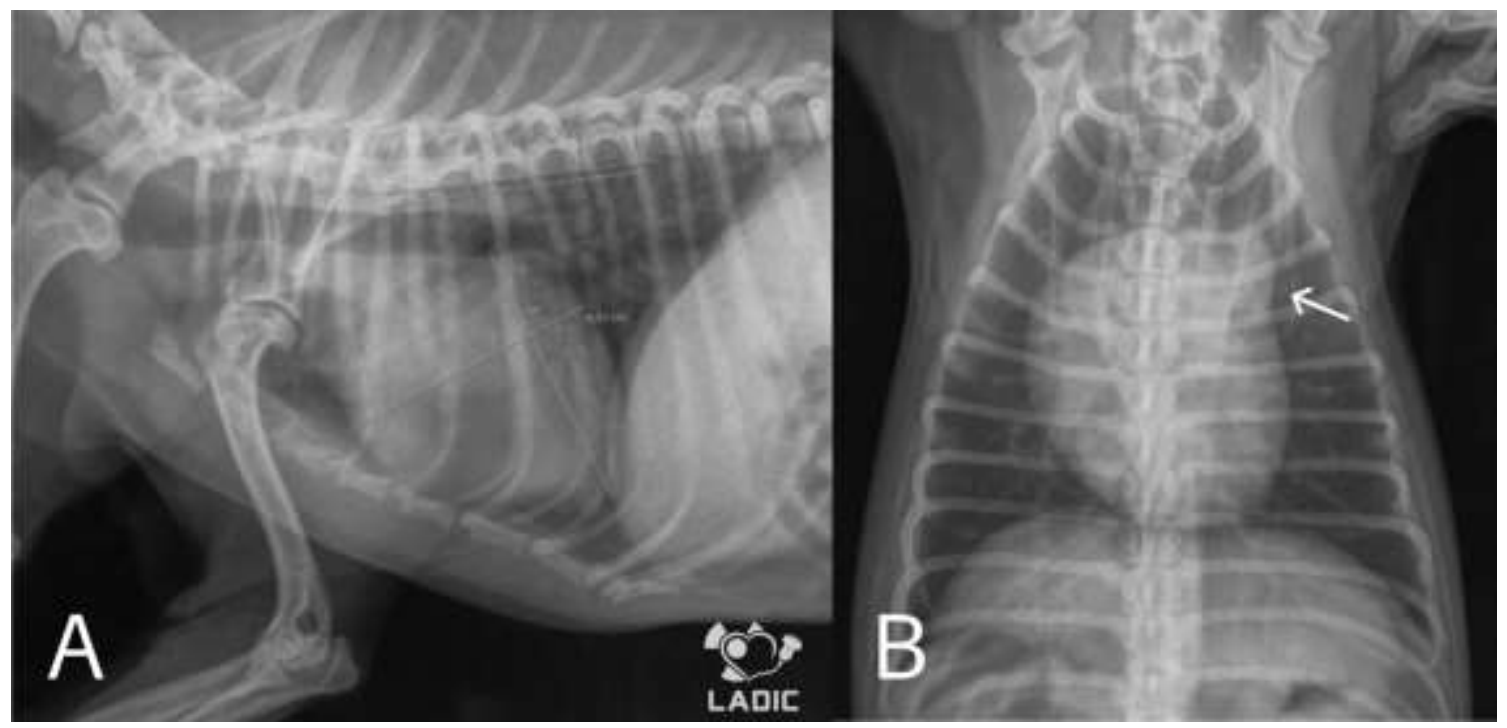

Fonte: LADIC. 
Figura 2: Radiografia em posição ventro-dorsal de uma cadela da raça Spitz Alemão (Caso 2) indicando aumento em região do tronco da artéria pulmonar (seta branca) e padrão pulmonar alveolar.

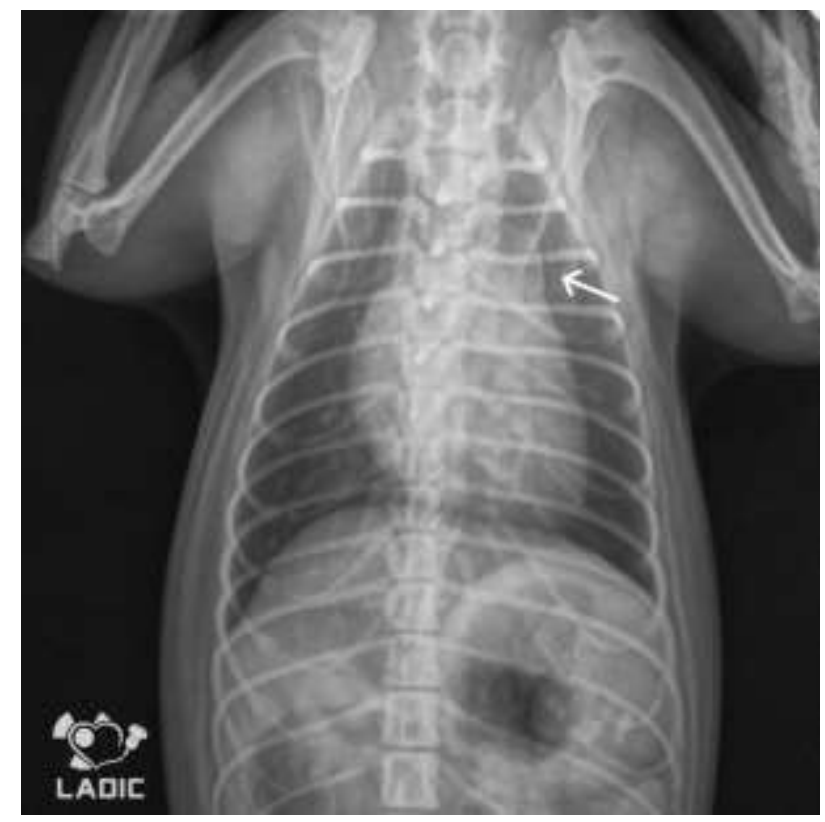

Fonte: LADIC.

Conforme o descrito por Godwin (2001), a ecocardiografia é considerada o método de eleição para o diagnóstico de EP, pois ele fornece informações sobre a estrutura e função cardíacas, e com o uso do Doppler, é possível determinar a velocidade do fluxo da artéria pulmonar e quantificar sua gravidade, além de determinar o tipo da estenose. Através deste exame, constatouse estenose do tipo valvar nas pacientes do presente estudo, ainda, no Caso 1, verificou-se comunicação interatrial concomitante a esta afecção. À ecocardiografia observou-se no animal do Caso 1 átrio esquerdo discretamente aumentado; ventrículo esquerdo com movimentação paradoxal septal aos modos bidimensional (modo-B) e movimento (modo-M); átrio direito aumentado; ventrículo direito aumentado com discreto aumento na espessura da parede; fechamento inadequado com a presença de refluxo discreto, ao Doppler em cores, na valva tricúspide; valva pulmonar com função e morfologia alterada, fechamento inadequado e fluxo sistólico aumentado com a presença de refluxo diastólico, turbulência após a região valvar (Figura 3) e presença de estrutura ecogênica subvalvar demonstrada na figura 4, presença de dilatação em tronco da artéria pulmonar (Figura 4), presença de comunicação interatrial do tipo forame oval medindo 0,26 com fluxo da esquerda para a direita. Na paciente do Caso 2 verificouse: aumento de átrio e da espessura da parede do ventrículo direito; fechamento inadequado com a presença de refluxo discreto, ao Doppler em cores, na valva tricúspide; e valva pulmonar com função e morfologia alterada, fechamento inadequado e fluxo sistólico aumentado com a presença de refluxo diastólico e turbulência após a região valvar (Figura 5). 
Figura 3: Imagem ecocardiográfica de uma cadela da raça Yorkshire Terrier demonstrando refluxo diastólico e turbulência após a região valvar (seta branca).

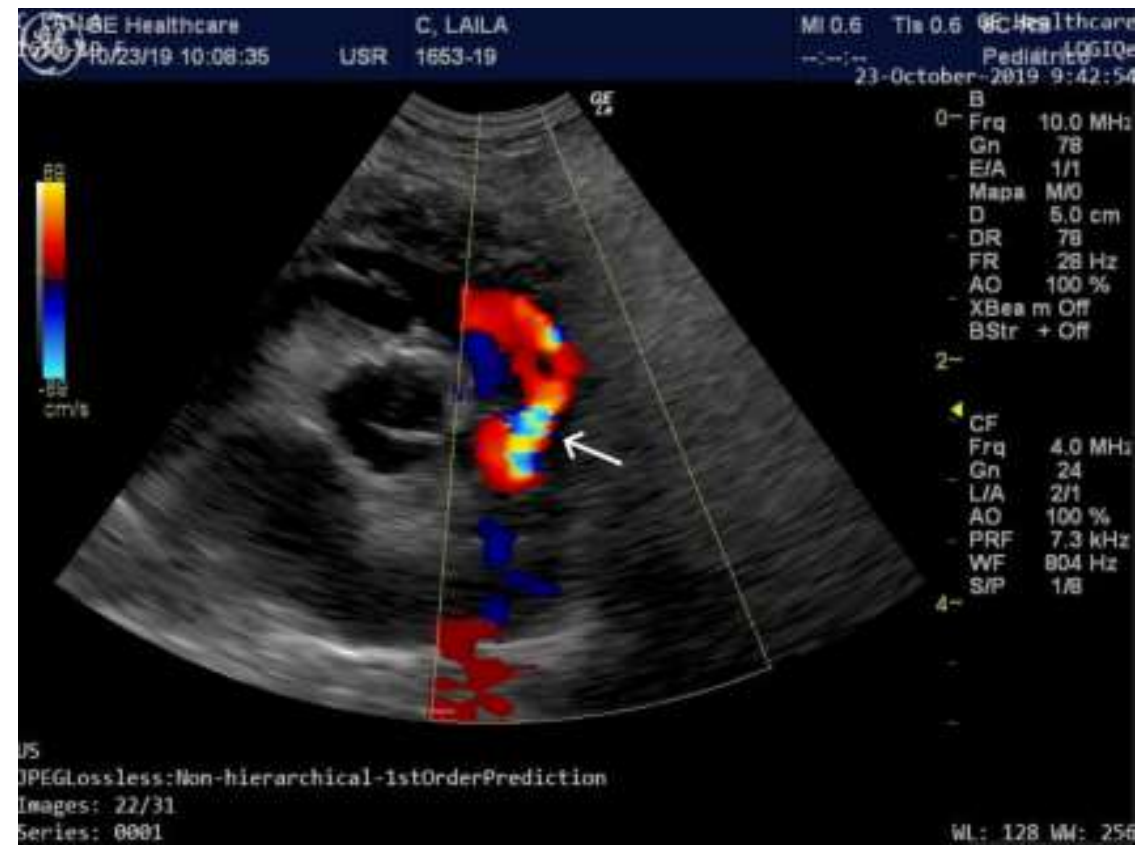

Fonte: LADIC.

Figura 4: Foto de imagem ecocardiográfica de uma cadela, da raça Yorkshire Terrier apresentando valva pulmonar com morfologia alterada e presença de estrutura ecogênica subvalvar (*) e dilatação em tronco da artéria pulmonar (seta branca).



Fonte: LADIC. 
Figura 5: Imagem obtida por ecocardiografia de um canino, da raça Spitz Alemão mostrando estenose pulmonar de origem valvar com refluxo diastólico e turbulência após a região valvar (seta branca).

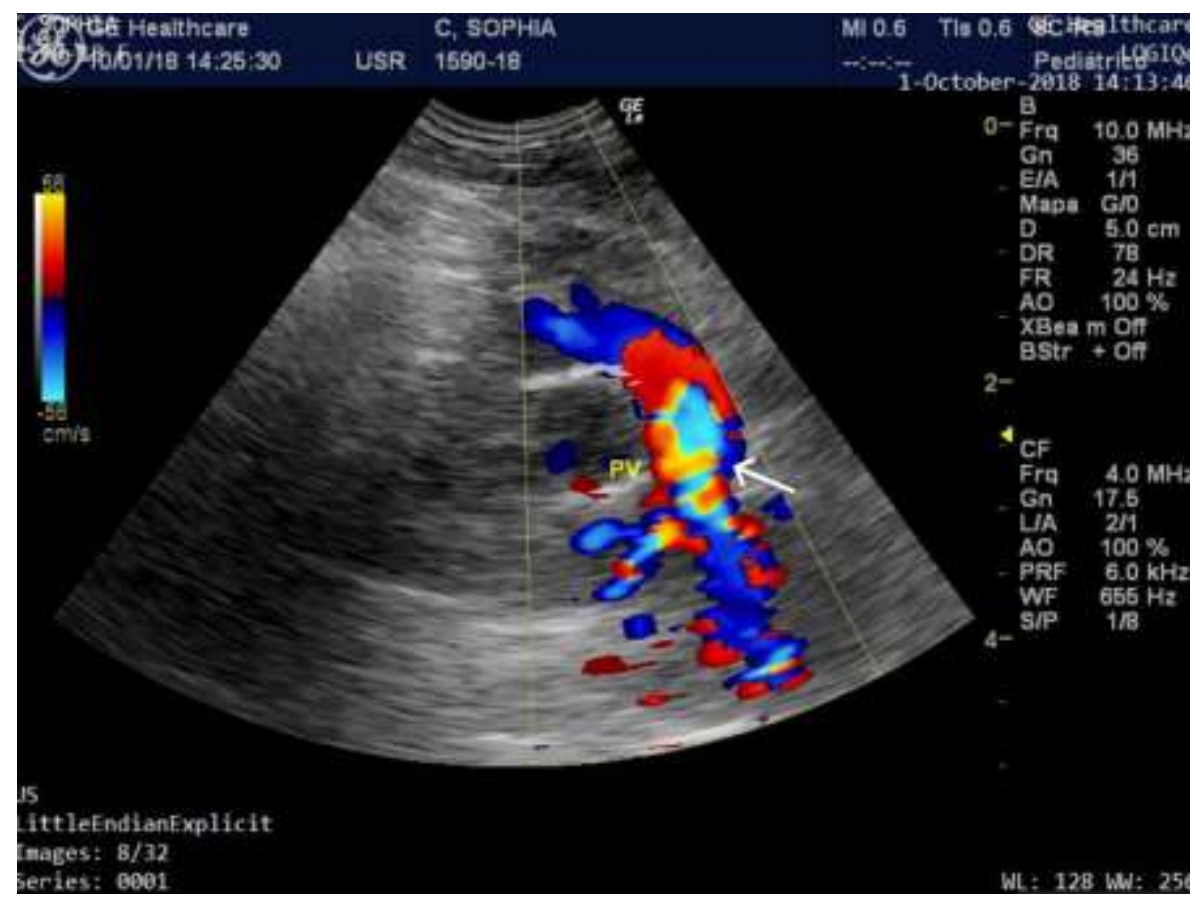

Fonte: LADIC.

A hipertensão arterial pulmonar (HPA) foi constatada na paciente 1 através das imagens dinâmicas do coração visualizadas no exame ecocardiográfico. A HPA moderada a grave pode apresentar alterações clássicas, tanto em modo-B como em modo-M, tais como a hipertrofia e dilatação ventricular direita, a dilatação da artéria pulmonar, o achatamento do septo com movimento paradoxal e o prolapso da válvula tricúspide e pulmonar (Escalda, 2014), sinais observados na paciente. Nesta paciente também foi visualizada comunicação interatrial, que é uma malformação cardíaca incomum em cães, de acordo com Cavalcanti et al. (2011), ocorrendo devido a um defeito no septo interatrial.

Para se estabelecer o tratamento para a EP, alguns critérios devem ser considerados, como severidade da estenose, magnitude do gradiente de pressão da válvula pulmonar mensurado, doenças cardíacas concomitantes (ou secundárias), e condição clínica geral do animal, dessa forma pode-se optar pelo tratamento clínico ou cirúrgico podendo, ainda, optar-se por não tratar animais assintomáticos (Schrope \& Kelch, 2007; Lázaro et al., 2015). No tratamento clínico são utilizados $\beta$ bloqueadores quando o gradiente de pressão pela valva for maior que $60 \mathrm{mmHg}$, sendo que esses fármacos auxiliam na redução da demanda de oxigênio pelo miocárdio com o aumento na perfusão coronária, em virtude da redução na frequência cardíaca e contratilidade. Efeitos estes, que ajudam a prevenir taquiarritmias e a reduzir a insuficiência do miocárdio ventricular direito, como também auxiliam na redução da obstrução dinâmica da via de saída do ventrículo direito (Silva Filho et al, 2011; Sousa et.al., 2020). Se o animal apresentar insuficiência cardíaca congestiva, o se tratamento é realizado através do uso de inibidores da enzima conversora de angiotensina e diuréticos (Filho et al., 2011; Lázaro et al., 2015). Em relação ao tratamento cirúrgico, a valvuloplastia por balão através de cateter percutâneo deve ser considerada como primeira opção (Bussadori et al., 2002; Brockmann, 2012; Lázaro et al., 2015). Optou-se pelo tratamento clínico nos casos relatados. O tratamento estabelecido para a paciente 1 foi a administração de Benazepril $(0,25 \mathrm{mg} / \mathrm{kg} / \mathrm{SID} / \mathrm{uso}$ continuo), um inibidor da enzima conversora de angiotensina, e Sildenafil (2mg/kg/BID/uso continuo), um fármaco inibidor da fosfodiesterase tipo 5 que atua no controle da hipertensão 
pulmonar, promovendo vasodilatação (Bach et al., 2006; Silva et al., 2012). Para o Caso 2, o tratamento clinico instituído foi a administração de Furosemida (1mg/kg/BID/5 dias), um diurético, e o $\beta$-bloqueador Atenolol (0,25 mg/kg/BID/30 dias).

Apesar dos critérios para estabelecimento do prognóstico nos animais portadores de obstruções da via de saída ventricular não estarem definidos, a literatura sugere nos casos de EP uma sobrevida normal, exceto quando há presença de defeitos concomitantes ou sinais clínicos de ICC (Oyama et al., 2005; Filho et al., 2011). Com isso, Oyama (2005) recomendouse a avaliação clínica periódica do paciente e realização de exames complementares, sendo assim, foi recomendado aos tutores das pacientes dos casos 1 e 2 o retorno em seis e doze meses, respectivamente. A paciente do Caso 1 realiza o tratamento prescrito há aproximadamente um ano e oito meses, segundo sua tutora, ela se apresenta bem fisicamente, porém não realiza a acompanhamento periódico como foi recomendado. A paciente do Caso 2 está em tratamento há dois anos e sete meses, neste período seu tratamento foi readequado, atualmente é administrado apenas Atenolol (0,25 mg/kg/BID/uso continuo), ela segue fazendo acompanhamento cardiológico e foi observado, no seu último exame ecocardiográfico, diminuição da estenose.

\section{Conclusão}

Embora a estenose pulmonar seja uma doença pouco frequente na rotina clínica, descrita principalmente em animais jovens e machos, o presente trabalho relata a afecção em duas fêmeas, sendo que uma delas apresentava idade maior que a esperada. Além disso, a presença de sinais clínicos compatíveis com doença cardíaca e sopros cardíacos auscultados nos exames específicos deve ser investigada também através de exames complementares de imagem, neste caso, o exame radiográfico apontou alterações compatíveis com a afecção, contudo foi o ecocardiograma o exame mais indicado para o diagnóstico definitivo.

Desta forma, conclui-se que a utilização do ecocardiograma para a avaliação morfofuncional cardíaca, e a avaliação dos fluxos sanguíneos através da ferramenta Doppler foi fundamental no diagnóstico de estenose pulmonar, sendo imprescindível no estabelecimento do tratamento adequado, reduzindo os sinais clínicos e melhorando o bem estar dos pacientes.

\section{Referências}

Bach, J. F., Rozanski, E. A., Macgregor, J., Betkowski, J. M. \& Rush, J. E. (2006). Retrospective evaluation of sildenafil citrate as a therapy for pulmonary hypertension in dogs. Journal of Veterinary Internal Medicine, 20(5), 1132-1135. https://doi.org/10.1111/j.1939-1676.2006.tb00711.x

Boon, J. A. (2011). Stenotic Lesions. In: Boon J. A. (2 ed.) Veterinary Echocardiography, 903-996.: Willey- Blackwell.

Bradley, K. (2013). Radiology of the Thorax. In: Holloway, A. \& McConnell, J. F. (ed.) BSAVA Manual of Canine and Feline Radiography and Radiology, 109-175. Quedgeley, Gloucester, UK: British Small Animal Veterinary Association.

Brambilla, P. G., Polli, M., Pradelli, D., Papa, M., Rizzi, R., Bagardi, M., \& Bussadori, C. (2020). Epidemiological study of congenital heart diseases in dogs: Prevalence, popularity, and volatility throughout twenty years of clinical practice. PLoS One, 15(7). https://doi.org/10.1371/journal.pone.0230160

Brockman, D. J. (2012). Surgical therapy for canine congenital valvular pulmonic stenosis: when and how?. Journal of Small Animal Practice, 53, 87-8. https://doi.org/10.1111/j.1748-5827.2012.01182.x

Buchanan, J. (1992). Causes and prevalence of cardiovascular diseases. W. B. Saunders Company.

Bussadori, C., Amberger, C., Bobinnec, G., \& Lombard, C. (2000). Guidelines for the echocardiographic studies of suspected subaortic and pulmonic stenosis. Journal of Veterinary Cardiology, 2(2), 15-22. https://doi.org/10.1016/S1760-2734(06)70007-8

Bussadori, C., DeMadron, E., Santilli, R., \& Borgarelli, M. (2001). Balloon valvuloplasty in 30 dogs with pulmonic stenosis: Effect of valve morphology and annular size on initial and 1-year outcome. Journal of Veterinary Internal Medicine, 15(6), 553-558. https://doi.org/10.1111/j.1939-1676.2001.tb01590.x

Bussadori, C., Domenech, O., Longo, A., Pradelli, D. \& Bussadori, R. (2002). Percutaneous catheter-based treatment of pulmonic ste- nosis and patent ductus arteriosus in a dog. Journal of Veterinary Cardiology, 4(2), 29-34. https://doi.org/10.1016/S1760-2734(06)70036-4

Cavalcanti, G. A., Tatibana, L. S., Varaschin, M. S., Araújo, R. B., \& Val, A. P. (2011). Defeito de septo atrial em cão idoso. Arquivo brasileiro de medicina veterinária e zootecnia, 63(3), 503-507. https://doi.org/10.1590/S0102-09352011000200033

Detweiler, D., \& Patterson, D. (1965). The prevalence and types of cardiovascular disease in dogs. Annals of the New York Academy of Sciences, 127(1), 481. 
Escalda, J. T. L. (2014). Comparação de dois métodos ecocardiográficos na deteção de hipertensão pulmonar em cães. (Dissertação de mestrado integrado em medicina veterinária). Faculdade de Medicina Veterinária, Universidade de Lisboa, Lisboa, Lx., Portugal.

Estrada, A., \& Maisenbacher, H. (2015). Pulmonic Stenosis. In Bonagura J. \& Twedt D. C. (15 ed.) Kirk's Current Veterinary Therapy XV 2811-2822. St. Louis, MO, EUA: Elsevier Health Sciences/ Saunders.

Silva Filho, J. C., Jorge, P. S. \& Franco, R. P. (2011). Alterações eletrocardiográficas de um cão com estenose de valva pulmonar, antes e após terapia a base de Maleato de Enalapril e Atenolol. Acta Veterinaria Brasilica, 5, 92-99. https://doi.org/10.21708/avb.2011.5.1.2009

Fox, P. R., Moïse, N. S., Evans, H. E., \& Bishop, S. P. (1999). Cardiovascular Anatomy. In: Fox, P. R., Sisson, D. \& Moïse, N. S. (2 ed.) Textbook of Canine and Feline Cardiology, Principles and Clinical Practice 13-24. W.B. Saunders Company.

Goodwin, J. K. (2001). Manual of Canine and Feline Cardiology, W. B. Saunders Company.

Kittleson, M. K. \& Kienle R. D. (1998). Small Animal Cardiovascular Medicine. St. Mosby.

Kobayashi, K., Hori, Y. \& Chimura, S. (2014). Plasma N-Terminal Pro B-Type Natriuretic Peptide Concentrations in Dogs with Pulmonic Stenosis. Journal of Veterinary Medical Science, 76(6), 827-831. https://doi.org/10.1292/jvms.13-0554

Lázaro, M. A., Lagares, J. S., De Carvalho, R. O. A., \& Ferreira F. S. (2015). Estenose Pulmonar Subclínica em Cão- Relato de Caso. Medvep- Revista Científica de Medicina Veterinária- Pequenos Animais e Animais de Estimação, 13(43), 6-11.

Locatelli, C., Spalla, I., Domenech, O., Sala, E., Brambilla, P. G., Bussadori, C. (2013). Pulmonic stenosis in dogs: Survival and risk factors in a retrospective cohort of patients. Journal of Small Animal Practice, 54(9), 445-452. https://doi.org/10.1111/jsap.12113

MacDonald, K. A. (2006). Congenital heart diseases of puppies and kittens. Veterinary Clinics of North America- Small Animal Practice, 36(3), 503-531. https://doi.org/10.1016/j.cvsm.2005.12.006

Oliveira, L. P. (2015). Relato de caso: estenose pulmonar em cão (Trabalho de conclusão de curso). Faculdade de Agronomia e Medicina Veterinária da Universidade de Brasília, Brasília, Distrito Federal, Brasil.

Oyama, M. A. (2005). Congenital heart disease. In: Ettinger, S. J.; Feldman, E. C. (7 ed) Textbook of Veterinary Internal Medicine 972- 102. St. Louis, MO, EUA: Elsevier Health Sciences/ Saunders.

Ristic, J. M. E., Marin, C. J., Baines, E. A. \& Herrtage, M. E. (2001). Congenital pulmonic stenosis a retrospective study of 24 cases seen between 1990-1999. Journal of Veterinary Cardiology, 3(2), 13-19. https://doi.org/10.1016/S1760-2734(06)70015-7

Santos, N. M. S. (2019). Estudo retrospectivo de 47 casos de estenose pulmonar valvular em cães (Dissertação de mestrado integrado em medicina veterinária) Universidade Lusófona de Humanidades e Tecnologias, Lisboa, Lx., Portugal.

Schrope, D. P. \& Kelch, W. J. (2007). Clinical and echocardiographic findings of pulmonar artery stenosis in seven cats. Journal of Veterinary Cardiology, 9 , 83-89. https://doi.org/10.1016/j.jvc.2007.09.001

Serrano, G. N. S. S. (2012). Estenose pulmonar no cão (Dissertação de mestrado integrado em medicina veterinária) Universidade Lusófona de Humanidades e Tecnologias, Lisboa, Lx., Portugal.

Silva, A. C., Oberlender, G., Muzzi, R. A. L., Muzzi, L. A. L., \& Coelho, M. R. (2012). Hipertensão Pulmonar em Cães E Gatos: Revisão de Literatura. Revista Científica Eletrônica de Medicina Veterinária, 19,13.

Sisson, D. (2015). Pulmonic Stenosis. In: E. Côté (3 ed.) Clinical Veterinary Advisor, Dogs and Cats 866-886. Elsevier Health Sciences/ Saunders.

Sousa, M. P. D., Melo, W. G. G. D., \& Alves, F. R. (2020). Estenose da artéria pulmonar em cães: revisão de literatura. Revista Científica de Medicina Veterinária, 34, 9.

Ware, W. A. (2015). Cardiopatia Congênita. In: Nelson, R. W.; Couto, C. G. (5a ed) Medicina interna de Pequenos Animais 96-106. Rio de Janeiro, RJ, Brasil: Elsevier.

Zani, A., Becchetti, E, Leonardi, P., \& Sinatra, A. (2014). Persistent left cranial vena cava draining into the left atrium associated with pulmonary stenosis in a French bulldog. Journal of Veterinary Cardiology, 16, 121-125. https://doi.org/10.1016/j.jvc.2014.03.003 